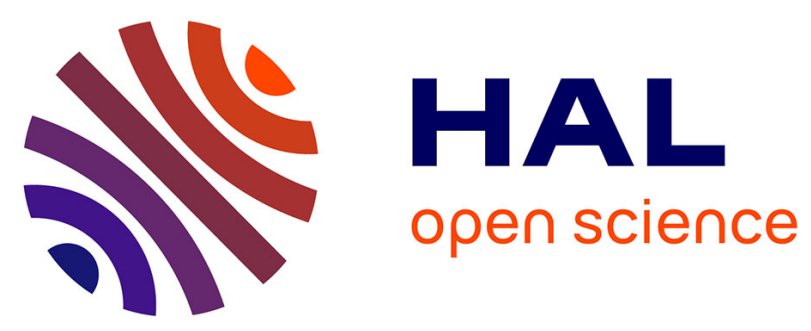

\title{
Comparison of Moment-Based Methods for Representing Droplet Size Distributions in Supersonic Nucleating Flows of Steam
}

\author{
Ali Afzalifar, Teemu Turunen-Saaresti, Aki Grönman
}

\section{- To cite this version:}

Ali Afzalifar, Teemu Turunen-Saaresti, Aki Grönman. Comparison of Moment-Based Methods for Representing Droplet Size Distributions in Supersonic Nucleating Flows of Steam. 16th International Symposium on Transport Phenomena and Dynamics of Rotating Machinery, Apr 2016, Honolulu, United States. hal-01891315

\section{HAL Id: hal-01891315 \\ https://hal.science/hal-01891315}

Submitted on 9 Oct 2018

HAL is a multi-disciplinary open access archive for the deposit and dissemination of scientific research documents, whether they are published or not. The documents may come from teaching and research institutions in France or abroad, or from public or private research centers.
L'archive ouverte pluridisciplinaire HAL, est destinée au dépôt et à la diffusion de documents scientifiques de niveau recherche, publiés ou non, émanant des établissements d'enseignement et de recherche français ou étrangers, des laboratoires publics ou privés. 


\title{
Comparison of Moment-Based Methods for Representing Droplet Size Distributions in Supersonic Nucleating Flows of Steam
}

\author{
Ali Afzalifar ${ }^{1 *}$, Teemu Turunen-Saaresti ${ }^{2}$, Aki Grönman ${ }^{3}$
}

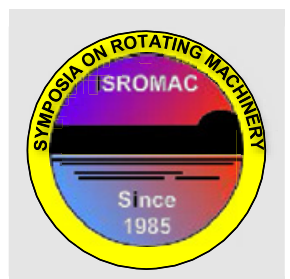

ISROMAC 2016

International

Symposium on Transport

Phenomena and Dynamics of Rotating Machinery

Hawaii, Honolulu

April 10-15, 2016

\begin{abstract}
This article investigates the effectiveness of two moment-based methods along with a monodispersed model (Mono) in representing the droplet size distribution and characteristics of steam flows with spontaneous condensation in supersonic nozzles. The moment-based methods are a conventional method of moments (MOM) along with its enhanced version using Gaussian quadrature, namely the quadrature method of moments (QMOM). The predictions of the droplet size distribution by these models are evaluated against the full spectrum resolved by an Eulerian-Lagrangian (E-L) method which tracks the evolution of the liquid phase in a Lagrangian frame of reference. On the other hand, an Eulerian reference frame is chosen to cast all the equations governing the phase transition and fluid motion for the MOM, QMOM and Mono. This choice of reference frame is essential especially to draw a meaningful comparison regarding complex flows in wet-steam turbines. The reason is that the most important advantage of the moment-based methods is that the moment-transport equations can be conveniently solved in an Eulerian frame avoiding burdensome challenges in working with a Lagrangian framework for complicated flows. The non-realizability problem and associated restrictions on temporal and spatial discretization schemes are discussed. The main focus is on the accuracy of the QMOM and MOM in representing the water droplet size distribution. The comparisons between models are made for two supersonic low-pressure nozzle experiments reported in the literature. Results show that the QMOM, particularly inside the nucleation zone, predicts moments closer to those of the E-L method. Therefore, for the test case in which the nucleation is significant over a large proportion of the domain, the QMOM clearly provides results in better agreements with the E-L method in comparison with the MOM.

\author{
Keywords \\ Steam-Nucleation —Droplet size distribution —QMOM-MOM-Eulerian-Lagrangian \\ 1, 2 \& 3 School of Energy Systems, Lappeenranta University of Technology, Lappeenranta, Finland \\ *Corresponding author: ali.afzalifar@lut.fi
}

\section{INTRODUCTION}

The realization of undesirable effects of wetness formation on the performance of steam turbines almost goes back to the era when these machines were utilized for the first time in the power industry [1]. Nonetheless, it is still challenging to implement numerical models which accurately take into account of the details of the liquid formation process. The weaknesses of the numerical models along with deficiencies in theories governing the phase transition make steam turbine manufacturers still rely on empirical methods. One of the details in the formation of wetness is the size of water droplets which greatly influences the flow field, subsequent nucleation processes, efficiency and erosion in the stages downstream of the primary nucleation sites [2] [3]. The presence of water droplets in different sizes (radii) causes the modeling methodology of wet-steam flows to fall under the category of polydispersed two-phase flows. In addition, the range of droplet radii is inherently continuous and wide in steam turbines. Thus, it is computationally very demanding and complicated to retain this type of information for droplets originating from different locations in the wet-steam turbines.

For a long time, the common practice was to employ the Eulerian-Lagrangian (E-L) method to preserve the droplet size distribution [4] [5] [6]. In this method, the Eulerian frame is used to numerically solve the motion equations related to the steam and the Lagrangian frame is employed to track the generation, and growth/decay of the water droplets along the streamlines and store the size distributions in a discrete fashion. The E-L method becomes very infeasible for modeling complex flows such as those in full-scale wet-steam turbines with multiple stages. The reason is that estimating the streamlines (or 
path lines for unsteady flows) for Lagrangian integration and then calculating the changes and reallocation of properties to the domain cells become extremely complicated in wet-steam turbines [7]. Consequently, the current commercial and in-house codes usually only apply the fully-Eulerian monodispersed methods (Mono) [8] [9] [10]. However, the Mono cannot retain information on the droplet size distribution. In contrast to the E-L method, the liquid phase is represented in the form of droplets with a single averaged radius.

Recently, the application of the method of moments (MOM) to wet-steam flows has received more attention [11] [12] [13]. The MOM is based on tracking the moments of droplet size distribution to predict wet-steam flows in turbines and it can be performed by applying an Eulerian reference frame as well as a Lagrangian one. The MOM can be enhanced to allow more complicated droplet growth equations through approximating discrete radii and weights by $n$-point Gaussian quadrature. The enhanced approach is named the quadrature method of moments (QMOM) which, much like the E-L respects the polydispersed nature of the wet-steam flows. Furthermore, it seems to be promising in modelling fullscale cases, as it conveniently lends itself to an Eulerian frame [7].

The application of the QMOM in modeling steam condensing flows is still in its infancy and, to the knowledge of the authors, it has not yet been employed for simulating full-scale wet-steam turbines. Furthermore, it is noticed that all the comparative studies between the moment-based and E-L methods were performed using a Lagrangian reference frame for both methods [14] [12]. However, the accuracy of the QMOM and MOM should be tested using an Eulerian framework, as the main advantage of these methods over the E-L method is that they can be cast in an Eulerian frame while preserving the information about the droplets size distribution. This article (in sections 1 and 2) briefly describes the main constituents of numerical models of non-equilibrium wetsteam flows. Then, (in section 3) the essence of the E-L method, MOM, QMOM and Mono are presented. In section 4, firstly the two considered nozzle test cases are introduced and their flow characteristics are discussed. Secondly, the non-realizability problem and the corresponding restriction on applying high-order convection schemes to the moment fluxes are explained. Then, all four methods are compared in the nozzle test cases. It is shown that the MOM and QMOM predict similar size distribution if the nucleation is not the dominant process in the domain, as opposed to the postnucleation droplet growth process. In general, results provided by the QMOM, particularly in the nucleation zones, are closer to those of the E-L method than results obtained by the MOM. In terms of global parameters such as the pressure and mean droplet diameter, all the methods, except for the Mono, predict similar distributions.

\section{PHASE CHANGE PROCESS}

Almost all non-equilibrium models mimic the phase change based on the prediction of the new phase evolution in forms of two consecutive stages. These stages are defined by the nucleation and droplet growth processes. Initially, the condensation is favored by the nucleation process providing the first clusters of the liquid phase. Then, the phase transition is governed by the growth of supercritical droplets which ideally removes the supercooling and reestablishes the equilibrium. The term "supercritical" refers to the droplets larger than the critical radius $r^{*}$, which is calculated according to the theory developed by Gibbs. The critical radius is related to the surface tension $\sigma$ and the supersaturation $S$, being the ratio of the vapor pressure to the saturation pressure $S=$ $p_{v} / p_{s}$, as follows:

$$
r^{*}=\frac{2 \sigma}{\rho_{d} R T_{v} \ln (S)}
$$

where $\rho_{d}$ is the liquid density, $R$ is the specific gas constant and $T_{v}$ is the vapor temperature. The steady nucleation rate of the critical droplets per unit of volume is given by the expression of Becker and Döring [15] and Zeldovich [16] for the classical nucleation theory which is denoted by $J_{B D}$,

$$
J_{B D}=\frac{\rho_{v}^{2}}{\rho_{d}}\left[\frac{2 \sigma}{\pi m^{3}}\right]^{\frac{1}{2}} \exp \left(-\frac{4 \pi r^{* 2} \sigma}{3 k_{B} T_{v}}\right)
$$

where $\rho_{v}$ is the vapor density, $m$ is the molecular weight and $k_{B}$ is Boltzmann's constant. This expression is modified by the correction factor of Kantrowitz [17] and Courtney [18] to allow the non-isothermal effects and partial pressure of clusters larger than a monomer, respectively. It has been shown that these corrections along with Gyarmathy's droplet growth equation [19] modified by Young [20], can provide reasonably accurate results for low-pressure wet-steam flows. Therefore, for a homogenous fluid, the final form of nucleation rate $J$ reads

$$
J=\frac{1}{(1+\theta)} \frac{1}{S} J_{B D}
$$

in which $1 /(1+\theta)$ and $1 / S$ are the non-isothermal and partial pressure corrections, respectively.

The employed droplet growth equation is that of Gyarmathy revised by Young, which can also be tuned with an empirical factor to match the low-pressure nozzle measurements. The droplet growth equation is merely an equation for heat transfer between the droplet and the surrounding vapor generalized for a wide range of Knudsen number $K n$ from the free molecular to continuum regimes. This equation is written as, 


$$
\frac{d r}{d t}=\frac{\lambda_{v}\left(1-\frac{r^{*}}{r}\right)\left(T_{s}-T_{v}\right)}{\rho_{d} L r\left(1+3.78 \frac{K n}{P r_{v}}(1-\psi)\right)}
$$

in which $\lambda_{v}$ is the thermal conductivity of the vapor, $r$ is the droplet radius, $T_{S}$ is the saturation temperature, $L$ is the specific enthalpy of evaporation, $P r_{v}$ is the vapor Prandtl number and finally $\psi$ is the empirical tuning factor proposed by Young. Equations 1-3 are used to formulate the source terms for the liquid phase, which are integrated in time to update the number and size of water droplets.

\section{FLOW EQUATIONS}

Neglecting the relative acceleration between the phases for a uniform pressure field, the transport equations for the mixture of droplets and vapor take the form of a single phase fluid. The assumption of no velocity slip between the droplets and vapor is justified in most cases especially for the extremely small droplets (much smaller than one micro meter) which originate from the homogenous nucleation and have a negligible inertial relaxation time. Thus, the flow equations of the mixture for an inviscid one-dimensional case in a domain with the cross-sectional area $A$ can be written as

$$
\frac{\partial}{\partial t}\left[\begin{array}{c}
\rho_{m} A \\
\rho_{m} u A \\
\rho_{m} E_{m} A
\end{array}\right]+\frac{\partial}{\partial x}\left[\begin{array}{c}
\rho_{m} u A \\
\left(\rho_{m} u^{2}+p_{v}\right) A \\
\rho_{m} u H_{m} A
\end{array}\right]=\left[\begin{array}{c}
0 \\
p_{v} \frac{d A}{d x} \\
0
\end{array}\right]
$$

where the subscript $m$ refers to the mixture variables, $u$ is the velocity, $E$ is the total internal energy and $H$ is the total enthalpy. If the wetness fraction $Y$ is known by neglecting the volume occupied by the liquid water droplets, the mixture variables are related to the vapor and liquid variables as below

$$
\begin{gathered}
\rho_{m}=\frac{\rho_{v}}{1-Y} \\
E_{m}=Y E_{d}+(1-Y) E_{v} \\
H_{m}=Y H_{d}+(1-Y) H_{v} .
\end{gathered}
$$

As the behavior of steam in the most practical conditions of steam turbines differs from the ideal gas assumption, the steam pressure and its thermodynamic properties are calculated using the Vukalovich equation of state [21]. The saturation pressure as a function of temperature is given according to an empirical equation reported in [22]. To keep the thermodynamic inconsistency between the liquid and the vapor properties to the minimum, the liquid enthalpy is calculated at the saturation temperature employing the ClausiusClapeyron relation. In addition, the liquid water density and its planar surface tensions are given by the equations given in [23] and [20], respectively.

The discretized form of equation (5) is solved using AUSM (Advection Upstream Splitting Method) flux splitter scheme [24]. The main idea of the AUSM is that the convective flux, $\vec{F}_{I+1 / 2}$, on the left and right sides of a cell face is decomposed into two parts, namely the convective and pressure parts. The convective part is calculated based on the primitive variables $(\rho, u, E$ and $H)$ of either the left or the right side of the cell face, depending on the sign of a so-called advection Mach number $M_{I+1 / 2}$ at the cell face. The pressure part is calculated as the sum of a negative pressure and a positive pressure which are functions of the Mach numbers and pressures of the left and right sides of the cell face, respectively. Therefore, the convective flux can be formulated as,

$$
\vec{F}_{I+1 / 2}=\left(M_{I+1 / 2}\right)\left[\begin{array}{c}
\rho_{m} c \\
\rho_{m} c u \\
\rho_{m} c H_{m}
\end{array}\right]_{L / R}+\left[\begin{array}{c}
0 \\
p_{L}^{-}+p_{v_{R}}^{+} \\
0
\end{array}\right]
$$

where $c$ is the frozen speed of sound in the mixture and subscripts $L$ and $R$ refer to variables of the left and right sides of the cell face, respectively. To calculate the primitive variables on the left and right sides of the cell faces, the conservative variables are interpolated from the cell centers applying the MUSCL approach. Then, the primitive variables are calculated for the left and right sides using the interpolated conservative variables. For example, the density at the left and right sides of the east face for the $I^{t h}$ cell is given as,

$$
\begin{gathered}
\rho^{L}=\rho_{I}+\frac{1}{4}\left[\begin{array}{r}
(1+k)\left(\rho_{I+1}-\rho_{I}\right) \\
\left.+(1-k)\left(\rho_{I}-\rho_{I-1}\right)\right]
\end{array}\right. \\
\rho^{R}=\rho_{I+1}-\frac{1}{4}\left[\begin{array}{r}
(1-k)\left(\rho_{I+2}-\rho_{I+1}\right) \\
\left.+(1+k)\left(\rho_{I+1}-\rho_{I}\right)\right]
\end{array}\right.
\end{gathered}
$$

where $k$ is set to $1 / 3$ to provide the third-order upwind spatial discretization. In this work, when the third-order accurate discretization is applied, the MUSCL scheme is stabilized with the third-order limiter function of Koren [25] to remove non-physical oscillations.

\section{INTEGRATION OF LIQUID PHASE}

To close the conservation equations set, the wetness can be computed using different techniques. As mentioned earlier, the evolution of the liquid phase is integrated in the Lagrangian frame for the E-L method and in the Eulerian frame for the other three methods. 


\subsection{LAGRANGIAN FRAMEWORK}

The employed method is similar to the one presented in [6] where a wetness integration module, which is completely separated from the flow solver, calculates the evolution of the wetness along the streamline. This module only needs to receive the pressure distribution over the streamline and returns the wetness fraction distribution over the streamline. The continuous spectrum of droplet sizes is collapsed into a finite number of droplet bins thorough the streamline. However, the entire spectrum can also be represented by a monodispersed droplet which is calculated through an averaging method [26]. The formation of new droplets by nucleation is tracked along the streamline. If at any position the nucleation rate exceeds a threshold, say $10^{8}$ per unit of mass, a new group of droplets is introduced into the domain. Owing to the fact that physically an infinite number of droplet groups can be formed, a finite number of bins are used to combine groups falling within a same radius interval. The total wetness is the sum of the wetness fractions of all bins,

$$
Y=\sum_{i=1}^{K} Y_{i}=\sum_{i=1}^{K} \frac{4}{3} \pi \rho_{d} N_{i} r_{i}^{3}
$$

where $K$ is the total number of bins, $N_{i}$ is the number density of the droplets in the $i^{\text {th }}$ bin, and $r_{i}$ is the average radius for the $i^{\text {th }}$ bin. The contributions to the wetness fraction of each bin through the nucleation and growth processes are formulated as

$$
\frac{D Y_{i}}{D t}=\frac{4}{3} J \pi r^{* 3}+4 \pi r_{i}^{2} \frac{d r_{i}}{d t}
$$

where the first term on the left-hand side represents the material derivative of wetness, and the first term on the right-hand side of equation (13) is applied if the critical radius falls within the radius interval associated with the $i^{\text {th }}$ bin. The radius of each bin is updated by integrating the droplet growth the equation (4) using a third-order Runge-Kutta scheme while keeping the vapor properties constant. It should be noted that in [6] the equation (4) was directly integrated resulting in an implicit equation for the new droplet radius after the growth process. Then, this implicit equation was solved iteratively using the Newton-Raphson method. However, as this equation contains a logarithmic term, it is observed that its solution is very sensitive to the initial guess and prone to yield imaginary numbers. Therefore, here it is preferred to perform the growth integration numerically using the Runge-Kutta scheme.

To estimate the temperature after each integration increment, a relation between the changes of mixture enthalpy $h_{m}$ and the pressure must be defined. This relation is derived by considering the fluid particle as an isolated thermodynamic system [6]:

$$
\frac{D h_{m}}{D t}=\frac{1}{\rho_{m}} \frac{D p}{D t}
$$

\subsection{METHOD OF MOMENTS}

The method of moments for modeling the wet-steam flows was first suggested in [27]. This method is based on the introduction of a phase space and the droplet number density function (NDF) denoted by $f$. The phase space consists of three external dimensions, which are aligned with the Cartesian coordinate system $(x, y, z)$ and one (or more) internal dimension $r$. The number of droplets per unit mass of the mixture whose sizes range between $r$ and $r+d r$ is equal to $\int f d r$. Therefore, the total number of droplets is given by $\int f d V$, in which $d V=$ $d x d y d z d r$. Consequently, by neglecting the droplet coalescence and breakage the population balance of the dispersed phase is given as,

$$
\frac{\partial}{\partial t}\left(\rho_{m} f\right)+\nabla \cdot\left(\rho_{m} f u\right)+\frac{\partial}{\partial r}\left(\rho_{m} f u_{r}\right)=\rho_{m} J
$$

where $u_{r}$ is the so-called "drift velocity" aligned with the internal dimension $r$ in the phase space. In other words, this velocity is equivalent to the droplet growth rate $d r / d t$. Since the equation (15) formulates the continuity principle for the liquid phase in terms of the NDF. By introducing the $j^{\text {th }}$ moment of droplet size distribution where $\mu_{j}=$ $\int r^{j} f d r$, after some mathematical manipulations (see [7] for details), the equation (15) is rewritten to represent the moment-transport equations:

$$
\begin{aligned}
\frac{\partial}{\partial t}\left(\rho_{m} \mu_{j}\right)+\nabla \cdot & \left(\rho_{m} \mu_{j}\right) \\
& =\rho_{m} J r^{j}+j \rho_{m} \int r^{j-1} u_{r} f d r
\end{aligned}
$$

The above equation needs to be solved only for the first four moments to define all of the essential interfacial processes between the liquid and vapor phases. The reason is that these moments, i.e. from the zeroth moment to the third one, contain all of the physical information required to define the liquid phase. The zeroth moment $\mu_{0}=\int f d r$ gives the droplet number density, the first moment $\mu_{1}=\int r f d r$ is equal to the sum of all droplets radii per unit of mass. Finally, the second and third moments are proportional to the total droplet surface area and volume per unit of mass, respectively. The only problem in solving the moment-transport equations is that, as the function $f$ is not known, the integral on the righthand side of equation (16) cannot be directly solved for an arbitrary form of $u_{r}$. The conventional moment-based methods are forced to apply assumptions or certain forms of $u_{r}$ which allow an exact closure for the equation (16). For instance, it can be shown that the moment-transport equations are closed in a recursive manner if the growth rate can be formulated as 


$$
u_{r}=a+b r
$$

where $a$ and $b$ are only dependent on properties of the surrounding vapor and independent of $r$. In this work, as an example of the conventional moment-based methods, the growth rate is calculated for the surface-areaaveraged radius $r_{20}=\left(\mu_{2} / \mu_{0}\right)^{1 / 2}$. As a result, according to the equation (17) the growth rate equates to a value independent of the droplet size, i.e. $b=0$ and $u_{r}=a$. Thus, the equation (16) is closed as follows:

$$
\frac{\partial}{\partial t}\left(\rho_{m} \mu_{j}\right)+\nabla \cdot\left(\rho_{m} \mu_{j}\right)=\rho_{m} J r^{j}+j \rho_{m} a \mu_{j-1}
$$

where $a$ is the growth rate computed by the equation (4) for $r=r_{20}$. This method and its results will subsequently be referred to as MOM.

Treating the growth process based on an averaged droplet size leads to inaccurate growth rates considering the wet-steam flows in turbines, in which the radii typically span over a very broad range [7]. To avoid the mentioned issue, the QMOM was presented by McGraw in 1997 [28]. The QMOM approximate the integral on the right-hand side of the equation (16), as the summation of products of weights, $w_{i}$, and radii, $r_{i}$, which are obtained using the $n$-point Gaussian quadrature procedure. Thus, the moments take the form as,

$$
\mu_{j}=\sum_{i=1}^{n} r_{i}^{j} w_{i} .
$$

Consequently, the equation (16) is closed through approximating its right-hand side as,

$$
\rho_{m} J r^{j}+\rho_{m} j \sum_{i=1}^{n} r_{i}^{j-1}\left(u_{r}\right)_{i} w_{i}
$$

where, $\left(u_{r}\right)_{i}$ is calculated for $r_{i}$ using the equation (4).

To improve the accuracy of the QMOM in representing the size distribution, the quadrature degree $n$ can be increased. A higher quadrature degree means that the size distribution is represented in terms of more sets of radius and weight. Due to the fact that the number of moments and the corresponding transport equations which have to be solved is double the quadrature degree, i.e. $2 n$. Therefore, a compromise between the required computational power and accuracy should be considered. It is suggested in [29] that a quadrature degree of 3 can provide the desirable balance between the efficiency and accuracy. This implies that the equation (16) must also be solved for two more moments (fourth and fifth moments). In this work, the results obtained by this method are indicated by QMOM.

After deciding about the degree of quadrature, the other crucial step is the moment-inversion algorithm which calculates the elements of a so-called Jacobi matrix. Then, weights and radii are computed through solving the eigenvalue problem of the Jacobi matrix. The overall efficiency and stability of the code depends on the moment-inversion algorithm performance. A comparison between the product-difference and Wheeler's algorithms for finding the abscissas (radii) and weights using Gaussian quadrature can be found in [30]. Here, use is made of Wheeler's algorithm owing to its enhanced stability compared to the other ones, such as the productdifference algorithm.

\subsection{MONODISPERSED METHOD}

In terms of computational power, this method is the most inexpensive and simplest one compared to the previously described methods. The reason is that the transport of the liquid phase is tracked by means of only two conserved variables. In most cases and also in the current work, these two conserved variables are; the total number of droplets and volume per unit of mass. In fact, this method can be loosely characterized as a moment method in which information on only two moments is stored, thereby these two moments have to be related to each other through an average droplet size. Consequently, the growth rate is also calculated only for an average radius, here the volume-averaged radius. This method and its results will subsequently be referred to as Mono.

\section{RESULTS AND DISCUSSION}

As the current test cases are simplified to quasi-onedimensional flows, it is computationally feasible to avoid the averaging procedure for the E-L method. This requires the number of droplet bins to be equal to the number of time steps during the Lagrangian integration of the liquid phase. As a consequence, all of the information on the droplet size distribution is preserved with an accuracy limited only by the integration time step. It should be mentioned that, storing this significant amount of data on size distribution is neither recommended nor necessary, especially for larger cases, as the required memory significantly increases. However, here the aim is to resolve the full spectrum of the size distribution, as opposed to the computational efficiency, to provide reliable benchmark calculations. It should be mentioned that in all methods except for the E-L method the liquid transport equations are solved in an Eulerian frame of reference. This is because the main merit of these methods is the convenient implementation in an Eulerian frame considering the fact that using a Lagrangian frame becomes hopelessly impractical for unsteady, theredimensional and complex flows in wet-steam turbines.

To form the comparisons, Nozzle A of Moore's experimental study [31] and the experiment number 252 from the Moses and Stein nozzle [32] are selected. In the former case, the flow was provided through supplying superheated steam, with a stagnation pressure of $0.25 \mathrm{bar}$ and superheating degree of $17 \mathrm{~K}$ at the nozzle inlet. In the latter case, the inlet stagnation pressure and superheating 
degree were set to 0.4005 bar and $25.26 \mathrm{~K}$, respectively. For the specified inlet conditions, both nozzles were designed to deliver steady supersonic flows in the course of spontaneous condensation. The normalized heights of the nozzles, with respect to the throat height, are depicted in Figure 1 (top). It can be seen that the different nozzle shapes and inlet conditions result in quite distinct patterns of supersaturation and consequently phase transition, see Figure 1 (bottom). The throat height is denoted by $s$ and is equal to $0.063 \mathrm{~m}$ and $0.01 \mathrm{~m}$ for Nozzle A and Moses and Stein nozzle, respectively. Due to the small dimensions of the Moses and Stein nozzle relative to the boundary layer thickness for this case the effective area is used as suggested in their work.

Unfortunately, the geometry of the converging part of Moore's nozzle was not reported in his work, however as the diverging part was just a straight line, it is clear that the nozzle curvature was discontinuous at the throat. Therefore, the shape of the converging part of Nozzle A is approximated by a straight line as given in [33]. This discontinuity of the curvature at the throat strongly influences the pressure and also can be seen in form of a sudden jump in supersaturation at the throat. On the other hand, the transonic and supersonic sections of the Moses and Stein nozzle were a continuous part of a circular arc which leads to a very smooth increase in supersaturation.
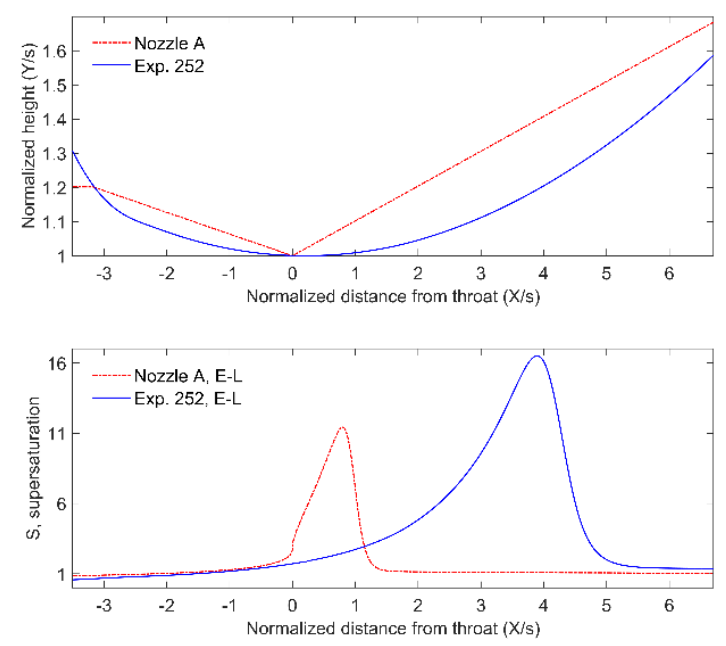

FIGURE 1. Normalized dimensions of nozzles (top) and distributions of supersaturation across the nozzles (bottom) obtained by the E-L method.

By the same token, the hypothetical expansion rates (according to the dry flow expansion) in these two nozzles are quite different. In Nozzle A the expansion rate peaks at approximately $3500 s^{-1}$ in the beginning of the nucleation zone and then would fall sharply to a very low value less than $2000 s^{-1}$. As a result of the low expansion rate and also small superheating degree at the inlet, for Nozzle A the nucleation zone occurs quite close to the throat and it is confined in a small proportion of the domain. This behavior can be seen in Figure 1 (bottom) which compares the distributions of supersaturation for these two nozzles in the presence of phase transition. On the contrary, the overall expansion rate in the Moses and Stein nozzle is quite significant and would gradually rise from $10000 s^{-1}$ to $13000 s^{-1}$ in the nucleation zone. Owing to the higher expansion rate and greater superheating degree at the inlet, for the Moses and Stein nozzle the nucleation zone is stretched more downstream of the throat. Thus, in general the nucleation process occurs comparatively gradual over a larger proportion of the domain until it can be augmented by higher supersaturation levels, compared to Nozzle A, see Figure 1 (bottom), to eventually reestablish the equilibrium.

\subsection{NON-REALIZABILITY PROBLEM}

Regardless of the employed moment-inversion algorithm, the correct calculation of radii and weights is unconditionally dependent on the realizability of the moment set. A realizable moment set corresponds to a (non-unique) NDF which is non-negative for all $r>0$. In fact, it is impossible to find any positive (or non-negative) NDF to describe a non-realizable moment set. The problem is that for a non-realizable moment set, some of the radii and weights may fall out of the physically acceptable range, or even often take negative values. The non-realizability problem arises from high-order advection or time-integration schemes [30]. More precisely, when conventional high-order spatial and temporal discretization schemes, such as the MUSCL, QUICK and Runge-Kutta schemes, are used to reconstruct the moment fluxes and update the moment set, the realizability of the new moment set cannot be guaranteed. The reason is that the mixing processes of moments from different spatial locations and time levels in the conventional high-order schemes treat each moment separately and therefore do not respect the interrelations between elements of the moment set. In fact, it was shown that only a first-order scheme in space along with the single-stage integration in time can guarantee the realizability of the updated moment set [34].

However, owing to the inherently excessive numerical diffusion of the first-order scheme, obtaining a gridindependent solution requires a very fine gird, and consequently, more computational power. As a result, some solutions to the non-realizability problem focus on developing new high-order advection schemes for which the realizability of the updated moment set can be guaranteed, these schemes are then termed as realizable schemes. For example, it is suggested in [35] and [36] to interpolate the radii and weights on the cell faces, instead of the moments themselves. Then, the moments at the cell faces are calculated using these interpolated radii and weights. However, the main difference compared to a conventional higher-order scheme is that, the radii are interpolated using the first-order scheme, while any highorder schemes are allowed for interpolation of the weights. A scheme built in this way is called a "quasi-higher-order scheme", since it does not recover the high-order accuracy 
in the interpolation of the radii. Another solution to derive a realizable high-order scheme is given in [37] by avoiding the complexity of the moment space through a transformation of moments into a canonical space and enforcing the realizability more conveniently in the canonical space. This solution offers a second-order scheme in both time and space.

The other type of solutions to the non-realizability problem tries to correct a corrupted moment set to recover the realizability condition instead of applying a realizable advection scheme. However, as it was suggested in [38], correcting moment sets should be avoided if it is possible to implement a realizable scheme. It was shown that moment-correction methods either are unsuccessful in recovering the realizability, or if they succeed, the corrected moment set can be quite different relative to the original one.

In this work, for both studied cases the MUSCL scheme results in non-realizable moment sets especially at the nucleation fronts where the very first droplets are generated in the domain and the moment gradients are very high. Therefore, for the MOM, QMOM and Mono, all the transport equations are solved using the first-order scheme with the single-stage explicit time integration. On the other hand, as for the E-L method the integration of the liquid phase is completely decoupled from the motion equations, this method is immune to the non-realizability problem. Therefore, the third-order MUSCL and RungeKutta schemes are used to enhance the integration in space and time, respectively.

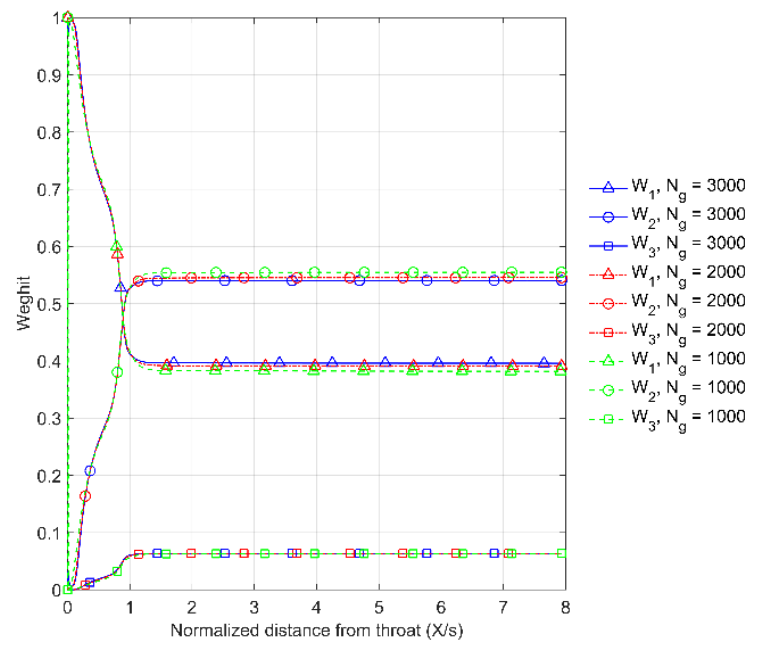

FIGURE 2. Comparison of weights from QMOM using grid sizes of 1000, 2000 and 3000 elements for Nozzle A; $N_{g}$ denotes the grid size.

The lack of flexibility in employing high-order advection schemes imposes a sever constraint on the flow solver for moment-based methods. Therefore, very fine grids must be used to compensate for the smoothing nature of the first-order scheme. In addition, all polydispersed methods using an Eulerian reference frame are notorious for a strong dependency on the grid size. Therefore, in the present article, very fine grids are used for both test cases to eliminate the uncertainties caused by the grid-sensitivity of solutions. Since it was shown in [38] that the weights and radii are much more sensitive to the grid size compared to the pressure and the averaged droplet size, these variables from the QMOM are considered for evaluating the grid-dependency. The distributions of weights and radii along Nozzle A are compared in figures 2 and 3 , respectively, using three different domain resolutions, i.e. domains with 1000, 2000 and 3000 elements. It can be concluded that for the domains with more than 2000 elements, results are quite independent of the grid size. Therefore, all of the results presented in this work are obtained for grids with 3000 elements, which are highly dense considering the simplified onedimensional test cases.

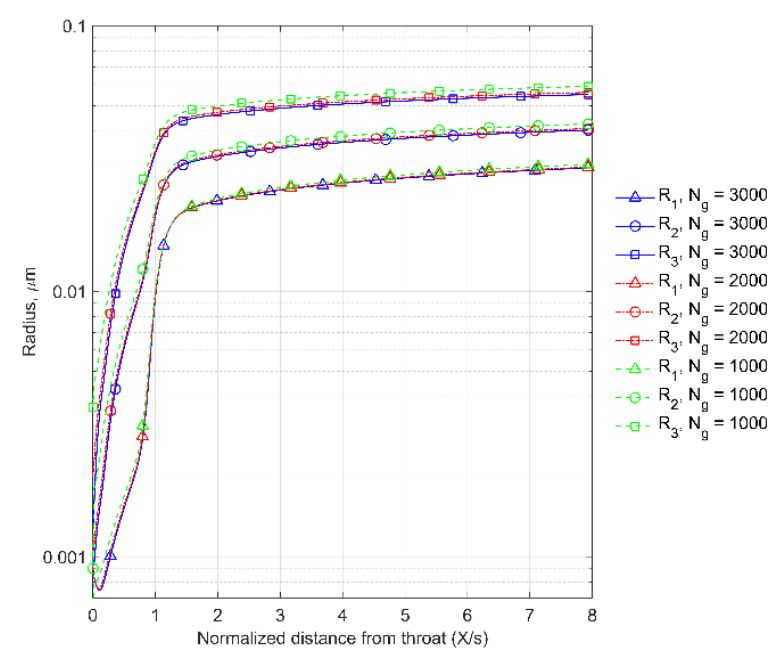

FIGURE 3. Comparison of radii from QMOM using grid sizes of 1000, 2000 and 3000 elements for Nozzle A; $N_{g}$ denotes the grid size.

Before discussing the results, it is noteworthy that although limited measurements for the pressure distributions and mean droplet sizes are available for both cases, it is preferred to base the comparisons on the E-L method as the benchmark results. In addition to the fact that the E-L method is arguably the most accurate among the considered methods, other reasons for this choice of basis for comparison are, firstly, the significant uncertainties over the measurements and also their interpretation methodology (especially for the droplet sizes) which are from more than several decades ago, secondly, the lack of measurements of other important variables such as the wetness fraction distribution, and thirdly, the inadequacies in theories defining the rates of nucleation and droplet growth, which can have combined effects in favor or against matching the measurements. 


\subsection{NOZZLE A}

By comparing the pressure and mean diameter distributions in figures 4 and 5 , respectively, we can see that all of the polydispersed models, i.e. the E-L method, MOM and QMOM, predict very similar profiles. In contrast, the Mono predicts slightly different values for the pressure and mean diameter distributions. However, considering the simplicity of the flow and phase transition process (triggered by a single nucleation event) in this test case, these slight differences can become significant

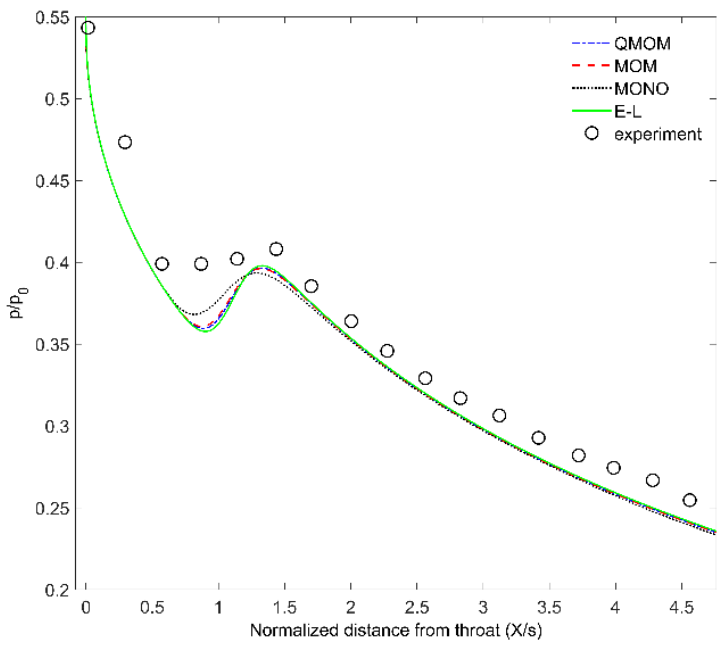

FIGURE 4. Comparison of pressure distributions in Nozzle A.

for practical problems. In particular, the Mono fails to accurately predict the correct location and properties of the Wilson point (for the two studied cases, the Wilson point can be interchangeably defined as the location of the lowest pressure, highest nucleation rate or highest supersaturation) relative to the other method.

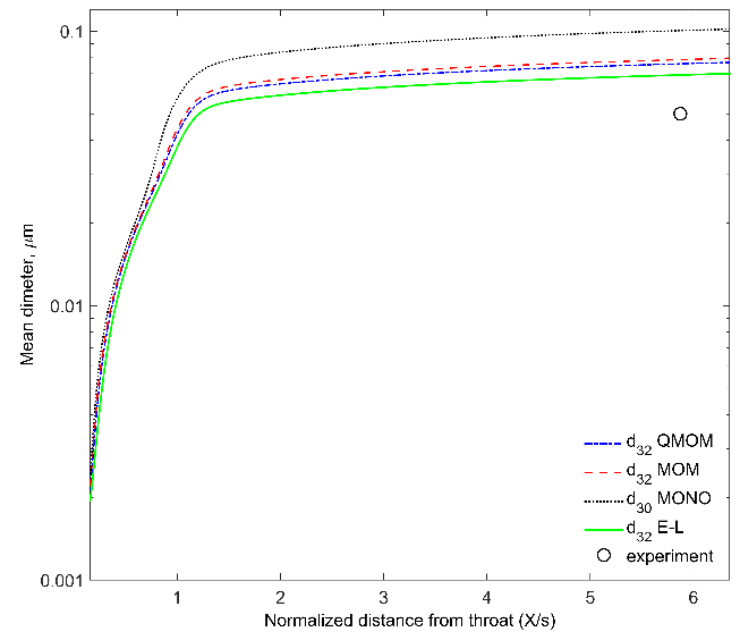

FIGURE 5. Comparison of droplet mean diameter distributions in Nozzle A.

Figure 6 shows the normalized distribution of moments over the nucleation zone which provides the best measure for comparing the characteristics of the size distributions given by the MOM and QMOM. It can be seen that, especially for moments of orders more than two, the dispersion of moments given by the MOM relative to the E-L method is more significant compared to the QMOM. The reason of this better agreement between the QMOM and the E-L method lies in the fact that the growth process is more accurately (and closely to the E-L method) modeled by the QMOM which benefits from three different radii compared to a single averaged radius $r_{20}$ in the MOM. Recalling section 3 , it should be noted that the fourth and fifth moments are neither required nor involved in the Interfacial processes using the MOM, and consequently do not affect the MOM results at all. However, here it is decided to update them during the MOM calculations only to draw a more informative comparison regarding the size distribution.

Moreover, since $\mu_{4}$ and $\mu_{5}$ are also available for the MOM, using Gaussian quadrature we can compute three radii and weights for this method as well. It is remarked that Gaussian quadrature approximates the NDF as the weighted sum of Dirac delta functions as shown below

$$
f \approx \sum_{i=1}^{n} w_{i} \delta\left(r-r_{i}\right) .
$$

Thereby, from the known moments the underlying size distribution can be inferred in a discrete form by the radii and weights. Figure 7 compares the radii and weights from the QMOM and MOM. As with Figure 6, it can be seen that the disagreement between the two methods is considerable in the nucleation zone. Nonetheless, after the place where the nucleation is quenched $(X / s \approx 1.2)$ the radii and weights from both methods become closer and stay in parallel with one another. It is reiterated that $\mu_{4}$ and $\mu_{5}$ do not influence the MOM results and the calculation of the radii and weights for the MOM is performed only once in the post-processing stage after obtaining the converged solution.

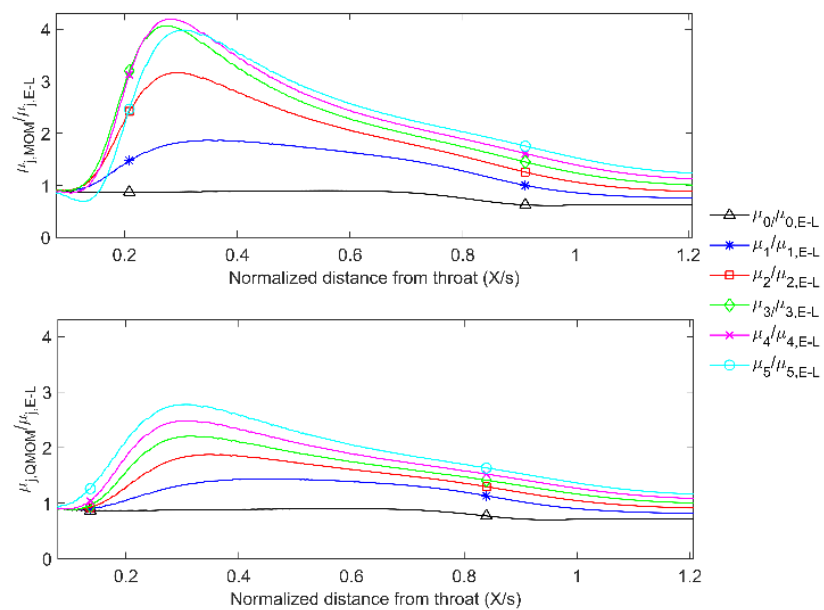

FIGURE 6. Distributions of normalized moments, relative to the $\mathrm{E}-\mathrm{L}$, of MOM (top) and QMOM (bottom) over the nucleation zone in Nozzle A. 
The last comparison investigates the agreement between the discrete approximations of size distribution by the moment-based methods and the full spectrum resolved by the E-L method. Figure 8 illustrates the $P$ distributions of the normalized weights over the radii given for these polydispersed methods at three locations being inside, just after and far downstream of the nucleation zone. As it is expected, the QMOM clearly outperforms the MOM inside the nucleation zone $(X / s=$ 0.3 ) and predicts a closer size distribution to that of the $\mathrm{E}-\mathrm{L}$ method. Downstream of the nucleation zone, predictions of both the QMOM and MOM are rather similar. In general, for wider distributions after the nucleation process $(X / s=1.5$ and 7.0) the discrete distribution by means of only three points cannot provide a tangible picture for comparison. It is worth mentioning that this matter does not necessarily indicate a wrong prediction of moments over the post-nucleation region, as Figure 6 shows that after $X / s=1.2$ the relative moments are very close to unity. Instead, it only indicates that one needs more sets of radii and weights to mimic the continuous distribution more clearly. After all, it can be argued that the QMOM bears slightly closer resemblances to the E-L method in Figure 8.

Interestingly, once the size distribution matures after the nucleation is stopped, in the absence of a secondary nucleation or evaporation it will retain its shape and only drift along the $r$ dimension due to the growth process, as it can be seen in figures 7 and 8 .
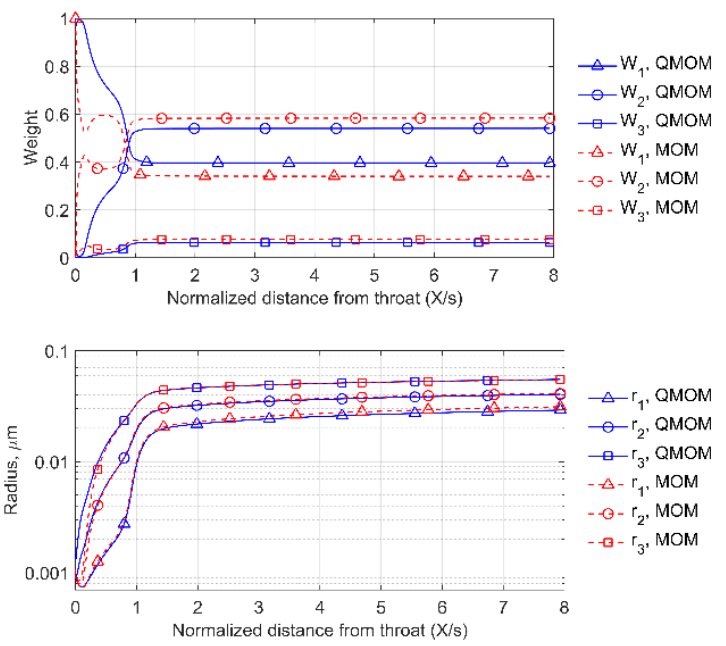

FIGURE 7. Comparison of distributions for weights (top) and radii (bottom) between QMOM and MOM, in Nozzle A.

As the equilibrium state reestablishes, after quenching the nucleation, the growth rates become almost independent of the droplet size, see Figure 9. Thus, the differences between the radii and weights from the MOM and QMOM remain unchanged. In plain English, the equation (4) in the absence of enough supersaturation can be nearly defined by a variable only dependent on the vapor properties. Hence, as discussed earlier for the equation (17), the QMOM reduces to the MOM in the post-nucleation region. Whether this behavior of the growth rate given by the equation (4) for the quasiequilibrium conditions in post-nucleation region is physically realistic or not is beyond the scope of the present work. For this case, the important observation is that the limitation of the nucleation zone (non-equilibrium region) to a very small part of the nozzle and the dominance of the post-nucleation droplet growth process in the domain lead to similar predictions of the radii and weights by the QMOM and MOM. Nevertheless, for the latter method no radii and weights existed during the calculation and the growth rate was computed for the averaged radius $r_{20}$.
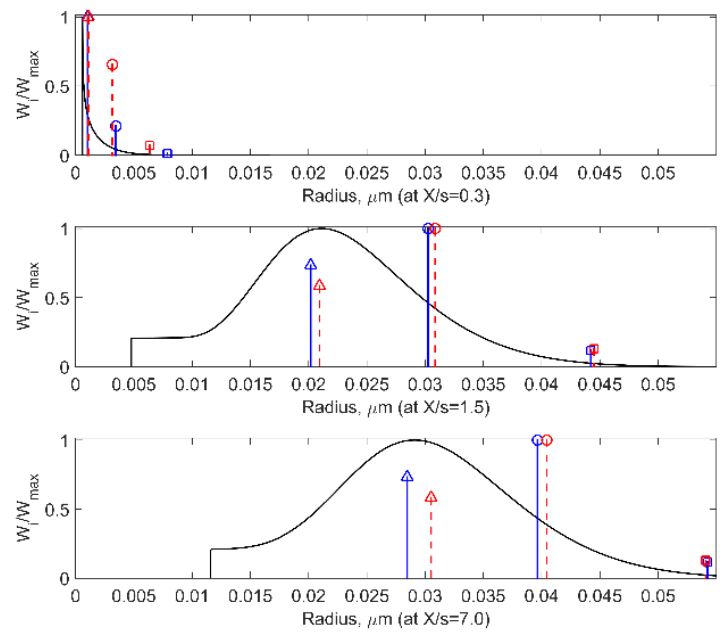

FIGURE 8. Comparison of discrete size distributions using Gaussian quadrature for QMOM and MOM with E-L at $X / s=$ 0.3 (top), $X / s=1.5$ (center) and $X / s=7.0$ (bottom).

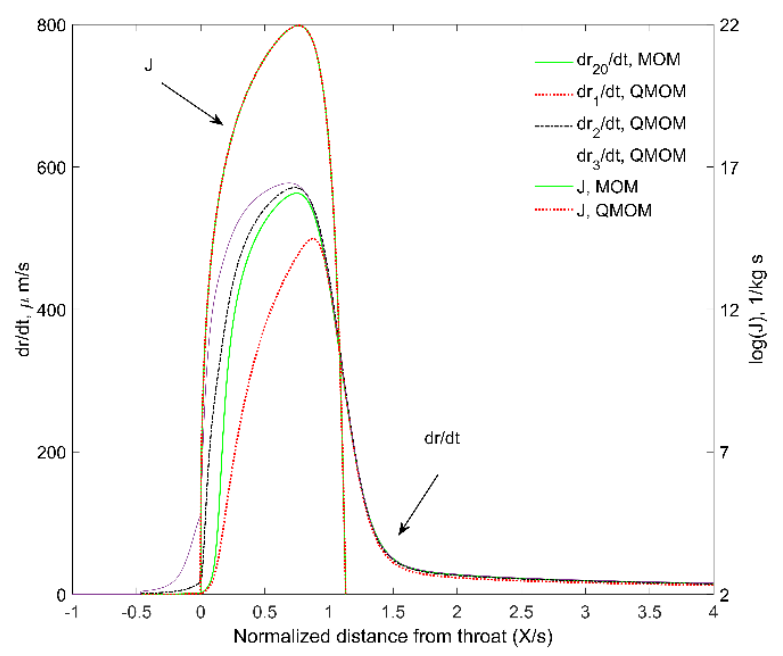

FIGURE 9. Distributions of nucleation rates and growth rates of droplet sizes present in calculations of QMOM and MOM in Nozzle A 


\subsection{EXPERIMENT 252 of MOSES AND STEIN}

As discussed in the beginning of this section, in the experiment 252 of Moses and Stein the nucleation process, or in general the non-equilibrium state, is more significant than the previous one and extends over a bigger proportion of the nozzle, see Figure 10 (cf. Figure 9). Figure 10 shows that the differences among the growth rates are larger and apparent over a much wider length of the domain compared to Nozzle A. For the same reason, Figure 11 shows that the deviation of the moments given by the MOM and QMOM from those of the E-L method is evident over a larger part of the nozzle which corresponds to the nucleation zone. However, in general the QMOM is in a closer agreement with the $\mathrm{E}-\mathrm{L}$ method compared to the MOM. In addition, Figure 12 illustrates more obvious disparities between the weights calculated for the QMOM and MOM. It can be seen that, in contrast with Nozzle A, even in the post-nucleation region the weights given by the MOM and QMOM are quite different. The wiggles appearing in the weights of both the MOM and QMOM, from the throat until $X / s \approx 1$, are related to the stability concerns over the momentinversion problem. The moment-inversion problem for calculating the radii (abscissas) and weights is prone to become ill-conditioned when the radii are very close to each other, which is the case for the experiment 252 , see the Figure 12 (bottom).

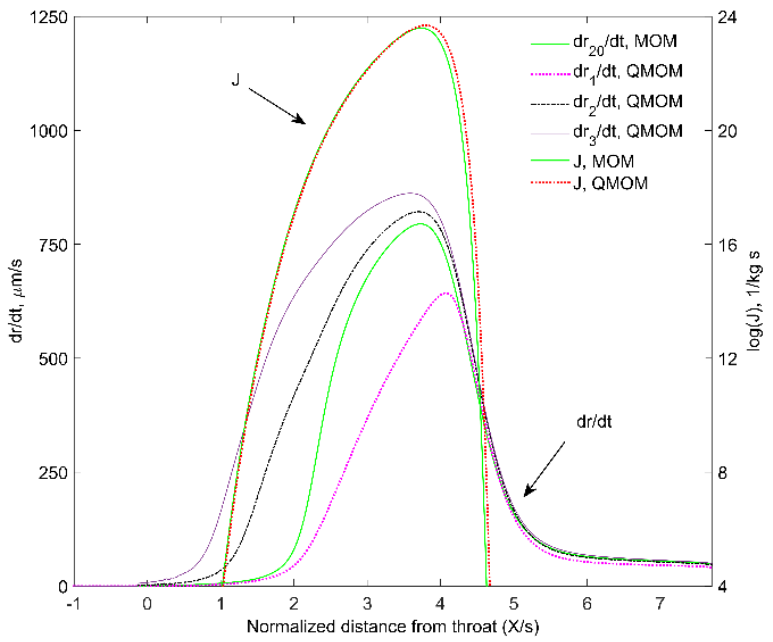

FIGURE 10. Distributions of nucleation rates and growth rates of droplet sizes present in calculations of QMOM and MOM in Exp. 252

Unlike the parameters related to the size distributions, and as with the previous case, all the polydispersed methods provide rather similar predictions for the pressure and mean dimeter distributions, see figures 13 and 14. However, it can be argued that for the pressure distributions the deviations between the moment-based methods and the E-L method and also between the QMOM and the MOM themselves are more noticeable compared to the Nozzle A.

\subsection{COMPUTATIONAL COST AND STABILITY}

The authors believe that a fair and constructive comparison of computational efficiency between methods differing in nature and architecture is not possible. In fact, this is the case for the E-L method compared to the other three which are quite alike. For instance, as in the E-L method the motion solution is decoupled from the integration of the liquid phase, it is possible to stabilize and accelerate the temporal integration by using the third-order explicit Runge-Kutta scheme, which allows CFL values larger than one. Moreover, the E-L method is naturally quite insensitive to the grid resolution, and as it is not affected by the non-realizability problem, employing highorder spatial discretizations are possible. Therefore, the E$L$ can provide grid-independent results on much coarser grids than required by the moment-based methods and consequently decreases the computational costs. However, for unsteady two/three dimensional flows, a Lagrangian framework might be completely inefficient.

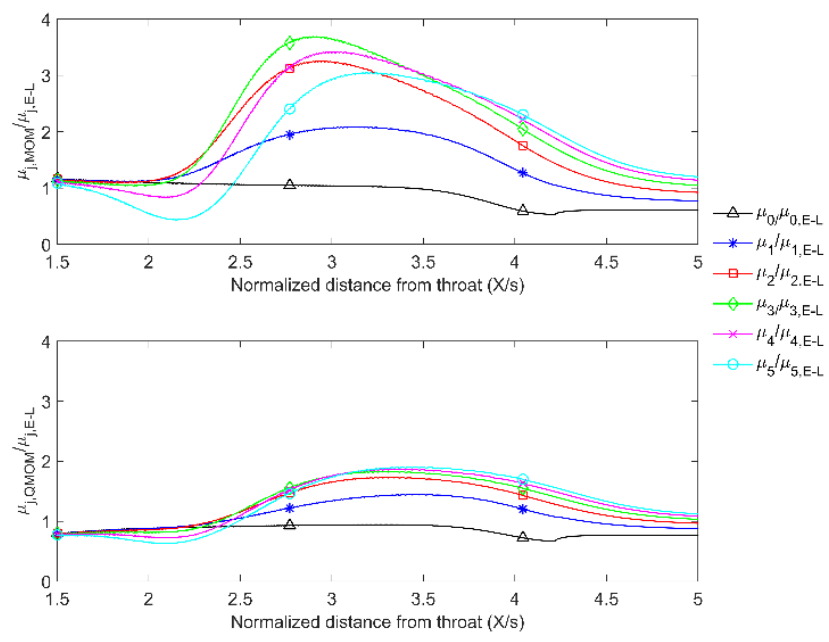

FIGURE 11. Distributions of normalized moments, relative to the E-L, of MOM (top) and QMOM (bottom) over the nucleation zone in Exp. 252.
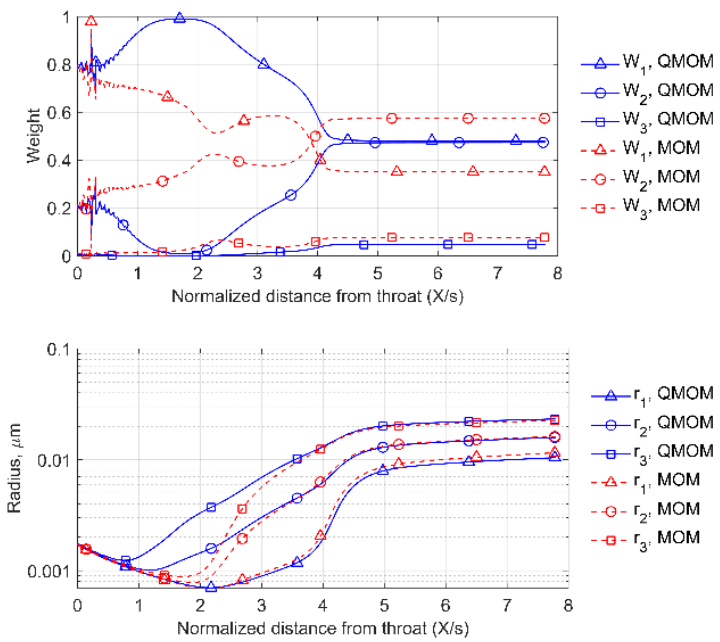

FIGURE 12. Comparison of distributions for weights (top) and radii (bottom) between QMOM and MOM, in Exp. 252. 
Fortunately, due to very similar structures of the MOM, QMOM and Mono, it is possible to draw a meaningful comparison in terms of CPU time and stability between them. Table 1 compares the CPU time of the MOM and the QMOM relative to that of the Mono. According to Table 1 the MOM is the most efficient method considering that its predictions for pressure and mean droplet size are very close to the QMOM and also E-L. However, as it is explained earlier, the MOM might incur considerable errors for cases with multiple nucleation events. As it is expected, Table 1 indicates that the QMOM is the most computationally expensive method in which the moment-inversion algorithm alone is responsible for a significant share of the increase in CPU time.

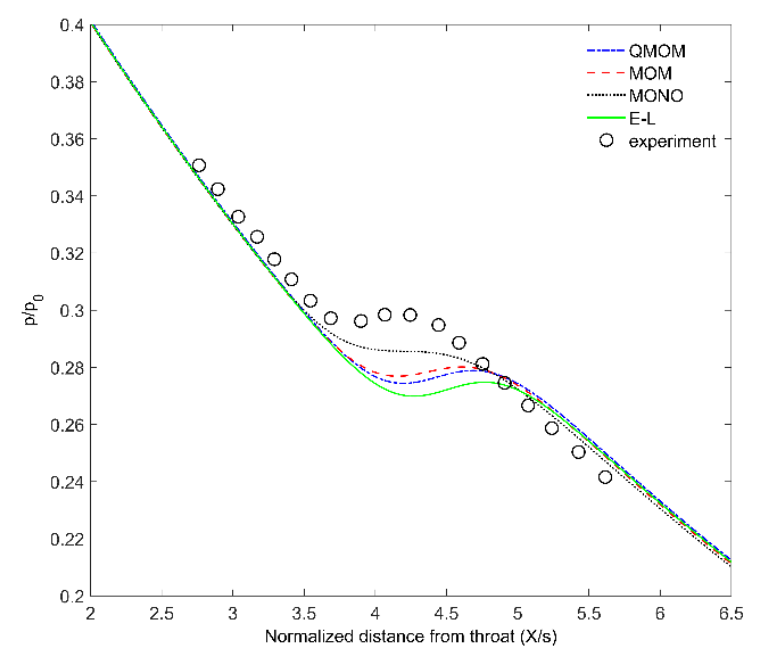

FIGURE 13. Comparison of pressure distributions in Exp. 252.

What is more, depending on the degree of quadrature the QMOM requires data on the higher-order moments (here $\mu_{4}$ and $\mu_{5}$ ) which for wet-steam flows take extremely small values. Therefore, it is necessary to store and treat the moments, especially during the moment-inversion procedure, using the double-precision format. This can considerably increase CPU time and memory for large cases. However, thanks to massive improvements in computational power, nowadays this problem does not seem insurmountable any more. In this work, calculations for all of the methods and variables are performed in double-precision format.

As discussed for the experiment 252, see Figure 12 (bottom), the moment-inversion problem becomes illconditioned for nearly monodispersed distributions. This issue is peculiar to the nucleation front of cases with almost uniform expansion rate in which superstation increases very slightly to initiate the nucleation, see Figure 1 (bottom). Therefore, in such conditions the size distribution at the early stages of the nucleation process has almost a zero spread and a location equal to the local critical droplet size. A remedy for such cases is to nucleate droplets belonging to a distribution with a suitable spread and a location equal to the local critical size.

\section{CONCLUSIONS}

The present article compares the ability of the MOM, QMOM and a monodispersed model to predict the droplet size distribution and flow behavior in the course of spontaneous condensation of steam. An EulerianLagrangian method is also applied as the basis of the comparisons in which the full spectrum of size distribution is resolved by tracking the liquid phase in a Lagrangian frame. Unlike the previous comparative studies, the solutions for the MOM, QMOM is obtained by means of an Eulerian framework. Since it is the feasible implementation of these methods in an Eulerian reference frame which make them attractive for modelling practical wet-steam flows.

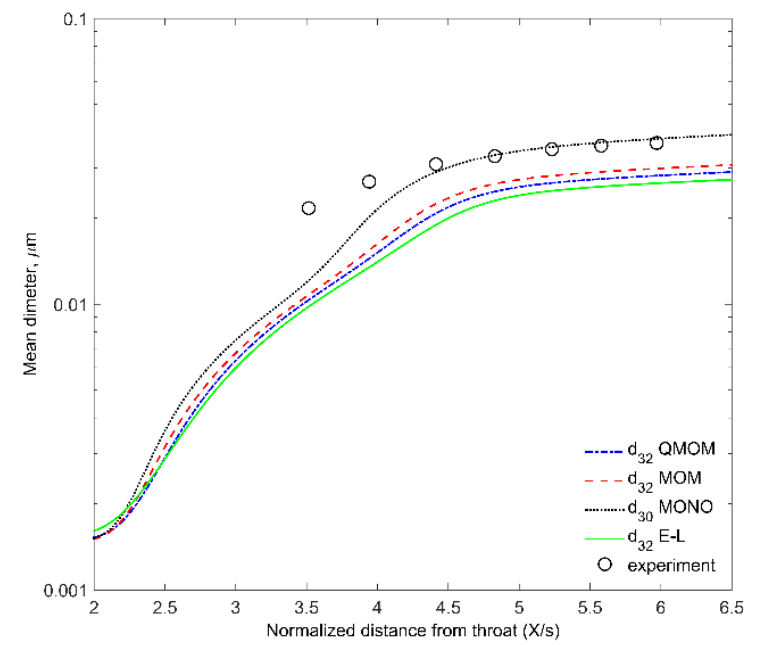

FIGURE 14. Comparison of droplet mean diameter distributions in Exp. 252.

TABLE 1. Relative CPU time of QMOM and MOM with respect to Mono for 100 time steps

\begin{tabular}{cccc} 
Grid size & MOM & QMOM & $\begin{array}{c}\text { Share of Wheeler's } \\
\text { algorithm in QMOM \% }\end{array}$ \\
3000 & 1.08 & 1.98 & $20 \%$ \\
1000 & 1.09 & 1.78 & $16 \%$ \\
\hline
\end{tabular}

The non-realizability problem with the moment-based methods and solutions available in the literature are discussed. It is remarked that for the moment-transport equations, this problem restricts the permissible advection schemes to the first-order accurate scheme, and therefore gives rise to the sensitivity of solutions to the grid size.

Two low-pressure supersonic nozzle experiments are used for comparison. Given the simplified onedimensional flows in these cases, it is concluded that the predictions of the pressure and mean diameter by the monodispersed model deviate considerably from the 
polydispersed ones. On the other hand, the three $L$ polydispersed methods, i.e. the MOM, QMOM and E-L $m$ method provide similar predictions for global variables $M$ such as the pressure and mean dimeter. Nevertheless, it $n$ is observed that the QMOM results in much closer $N$ agreements with the E-L method for the moments and $p$ estimation of size distributions especially in the $\mathrm{Pr}$ nucleation zone.

It is shown that the Gyarmathy's droplet growth $R$ equation (revised by Young) becomes almost $s$ independent of the droplet radius in the absence of $S$ enough supersaturation in the post-nucleation region. $T$ This behavior of the droplet growth equation reduces the $u$ QMOM to the MOM in a region with negligible supersaturation. Thus, judging based on the given weights and radii in the first test case in which the nucleation process is confined to a small part of the domain and the post-nucleation droplet growth covers a much larger length of the nozzle, it seems that the MOM and QMOM relate to similar underlying size distributions. On the other hand, for the second case in which the nucleation process is more significant and extends over a large proportion of the nozzle, the weights calculated for the MOM and QMOM are quite different even after the nucleation is quenched. Therefore, for wet-steam turbines in which flows undergo several consecutive nucleation processes, accurate predictions by the MOM cannot be expected, as the MOM possesses no ability (compared to the QMOM with an adjustable ability) to accommodate the required details of size distribution during the nucleation and growth processes. In the future, practical cases with several nucleation events must be considered to investigate how the accumulation of errors can deviate the MOM results from those of the QMOM, and whether a desirable trade-off between the computational costs of the QMOM and its performance is achievable.

\section{ACKNOWLEDGEMENTS}

The authors would like to thank the Academy of Finland for funding this work.

\section{NOMENCLATURE}

A

$b$

$c$

E

$f$

$\vec{F}$

$h, H$

$J$

$k$

$k_{B}$

$K$

Kn
Variable in the equation (17)

Cross-sectional area

Variable in the equation (17)

Frozen speed of sound

Total internal energy

Droplet number density function

Convective fluxes

Total and static enthalpy

Nucleation rate

Parameter in MUSCL scheme

Boltzmann's constant

Total number of droplet bins

Knudsen number
Specific enthalpy of evaporation

Molecular weight

Mach number

Degree of quadrature

Droplet number density of a droplet bin

Pressure

Prandtl number

Droplet radius

Specific gas constant

Throat height

Supersaturation

Temperature

Velocity

Volume in the phase space

Weight

Wetness fraction of a droplet bin

Streamwise coordinate

Wetness fraction or nozzle height

\section{GREEK LETTERS}

$\gamma \quad$ Specific heats ratio

$\theta \quad$ Non-isothermal correction parameter

$\lambda \quad$ Thermal conductivity

$\mu \quad$ Moment of distribution

$\rho \quad$ Density

$\sigma \quad$ Surface tension

$\psi \quad$ Empirical factor in equation (4)

\section{SUPERSCRIPTS AND SUBSCRIPTS}

$d \quad$ Liquid phase

$g$

$i$

Refers to the grid size

Radii and weights or droplet bin index

Computational cell index

Moment index

Mixture of vapor and liquid

Refers to $r$ dimension in the phase space

Vapor phase

Becker and Döring equation for $J$

Critical radius

Surface-area-averaged value

Volume-averaged value

Sauter mean value

\section{REFERENCES}

[1] M. J. Moore and C. H. Sieverding, Two-phase steam flow in turbines and separators, Washington: Hemisphere Publishing Corporation, 1976.

[2] A. White, J. Young and P. Walters, "Experimental validation of condensing flow theory for a stationary cascade of steam turbine blades," Philosophical Transactions of the Royal Society of London A: Mathematical, Physical and Engineering Sciences, vol. 354, no. 1704, pp. 5988, 1996. 
[3] F. Bakhtar, R. Henson and H. Mashmoushy, "On the performance of a cascade of turbine rotor tip section blading in wet steam. Part 5: theoretical treatment," Proceedings of the Institution of Mechanical Engineers, Part C: Journal of Mechanical Engineering Science, vol. 220, no. 4, pp. 457-472, 2006.

[4] S. A. Skillings and R. Jakson, "A robust 'timemarching' solver for one-dimensional nucleating steam flows," International journal of heat and fluid flow, vol. 8, no. 2, pp. 139-144, 1987.

[5] F. Bakhtar and M. M. Mohammadi Tochai, "An investigation of two-dimensional flows of nucleating and wet steam by the time-marching method," International Journal of Heat and Fluid Flow, vol. 2, no. 1, pp. 5-18, 1980.

[6] J. B. Young, "Two-dimensional, nonequilibrium, wet-steam calculations for nozzles and turbine cascades," J. Turbomachinery, vol. 114, no. 3, pp. 569-579, 1992.

[7] A. J. White and M. J. Hounslow, "Modelling droplet size distributions in polydispersed wetsteam flows," International Journal of Heat and Mass Transfer, vol. 43, no. 11, pp. 1873-1884, 2000.

[8] M. Mccallum and R. Hunt, "The flow of wet steam in a one-dimensional nozzle," International journal for numerical methods in engineering, vol. 44, no. 12, pp. 1807-1821, 1999.

[9] A. G. Gerber, "Inhomogeneous multifluid model for prediction of nonequilibrium phase transition and droplet dynamics," Journal of fluids engineering, vol. 130, no. 3, p. 031402, 2008.

[10] S. Dykas and W. Wróblewski, "Single-and twofluid models for steam condensing flow modeling," International Journal of Multiphase Flow, vol. 37, no. 9, pp. 1245-1253, 2011.

[11] D. A. Simpson and A. J. White, "Viscous and unsteady flow calculations of condensing steam in nozzles," International journal of heat and fluid flow, vol. 26, no. 1, pp. 71-79, 2005.

[12] A. G. Gerber and A. Mousavi, "Application of quadrature method of moments to the polydispersed droplet spectrum in transonic steam flows with primary and secondary nucleation," Applied mathematical modelling, vol. 31, no. 8, pp. 1518-1533, 2006.

[13] A. G. Gerber and A. Mousavi, "Representing polydispersed droplet behavior in nucleating steam flow," Journal of fluids engineering, vol. 129, no. 11, pp. 1404-1414, 2007.
[14] A. J. White, "A comparison of modelling methods for polydispersed wet-steam flow," International journal for numerical methods in engineering, vol. 57, no. 6, pp. 819-834, 2003.

[15] R. Becker and W. Döring, "Kinetische behandlung der keimbildung in übersättigten dämpfen," Annalen der Physik, vol. 416, no. 8, pp. 719-752, 1935.

[16] J. Zeldovich, "On the theory of new phase formation," Zh Eksp Theor Fiz, vol. 12, p. 525538, 1942.

[17] A. Kantrowitz, "Nucleation in very rapid vapor expansions," J. Chemical Physics, vol. 19, no. 9, p. 1097, 1951.

[18] W. G. Courtney, "Remarks on homogeneous nucleation," J. Chemical Physics, vol. 35, no. 6, pp. 2249-2250, 1961.

[19] G. Gyarmathy, "Grundlagen einer theorie der nassdampfturbine, Diss. Techn. Wiss. ETH Zürich, Nr. 3221, Ref.: W. Traupel, Korref.: J. Ackeret," 1962.

[20] J. B. Young, "Spontaneous condensation of steam in supersonic nozzles," PhysicoChemical Hydrodynamics (PCH), vol. 3, pp. 57-82, 1982.

[21] M. Vukalovich, Thermodynamic Properties of Water and Steam, sixth ed., Mashgis, Moscow, 1958.

[22] J.H. Keenan, F.G. Keyes, P.G. Hill, J.G. Moore, Steam Tables: Thermodynamics Properties of Water Including Vapor, Liquid and Solid Phases, New York: John Wiley \& Sons, 1978.

[23] J. B. Young, "An equation of state for steam for turbomachinery and other flow calculations," J. Engineering for Gas Turbines and Power, vol. 110, no. 1, pp. 1-7, 1988.

[24] M. S. Liou and C. J. Steffen, "A new flux splitting scheme," Journal of Computational physics, vol. 107, no. 1, pp. 23-39, 1993.

[25] B. Koren, "A robust upwind discretization method for advection, diffusion and source terms," Notes on numerical fluid mechanics, vol. 45, p. 117138, 1993.

[26] A. G. Gerber, "Two-phase Eulerian/Lagrangian model for nucleating steam flow," Journal of Fluids Engineering, vol. 124, no. 2, pp. 465-475, 2002.

[27] P. G. Hill, "Condensation of water vapour during supersonic expansion in nozzles," J. Fluid Mech, vol. 25, no. 3, pp. 593-620, 1966.

[28] R. McGraw, "Description of aerosol dynamics by the quadrature method of moments," Aerosol 
Science and Technology, vol. 27, no. 2, pp. 255265, 1997.

[29] D. L. Marchisio, J. T. Pikturna, R. O. Fox, R. D. Vigil and A. A. Barresi, "Quadrature method of moments for population-balance equations," AIChE Journal, vol. 49, no. 5, pp. 1266-1276, 2003.

[30] D. L. Marchisio and R. O. Fox, Computational models for polydisperse particulate and multiphase systems, Cambridge University Press, 2013.

[31] M. J. Moore, P. T. Walters, R. I. Crane and B. J. Davidson, "Predicting the fog-drop size in wetsteam turbines," in Wet Steam 4 Conference, Warwick, 1973.

[32] C. Moses and G. Stein, "On the growth of steam droplets formed in a Laval nozzle using both static pressure and light scattering measurements," Journal of Fluids Engineering, vol. 100, no. (3), pp. 311-322, 1978.

[33] M. J. Kermani and A. G. Gerber, "A general formula for the evaluation of thermodynamic and aerodynamic losses in nucleating steam flow," International journal of heat and mass transfer, vol. 46, no. 17, pp. 3265-3278, 2003.

[34] O. Desjardins, R. O. Fox and P. Villedieu, "A quadrature-based moment method for dilute fluid-particle flows," Journal of Computational Physics, vol. 227, no. 4, pp. 2514-2539, 2008.

[35] V. Vikas, Z. J. Wang, A. Passalacqua and R. O. Fox, "Development of high-order realizable finitevolume schemes for quadrature-based moment method," 48th AIAA Aerospace Sciences Meeting Including the New Horizons Forum and Aerospace Exposition , 2010.

[36] V. Vikas, Z. J. Wang, A. Passalacqua and R. O. Fox, "Realizable high-order finite-volume schemes for quadrature-based moment methods," Journal of Computational Physics, vol. 230, no. 13, pp. 5328-5352, 2011.

[37] D. Kah, F. Laurent, M. Massot and S. Jay, "Journal of Computational Physics," A high order moment method simulating evaporation and advection of a polydisperse liquid spray, vol. 231, no. 2, pp. 394-422, 2012.

[38] A. Afzalifar, T. Turunen-Saaresti and A. Grönman, "Non-realizability problem with quadrature method of moments in wet-steam flows and solution techniques," in ASME Turbo Expo 2016: Turbine Technical Conference and Exposition,, Seoul, South Korea, June 13-17, 2016, GT2016-57796, 2016.
[39] F. Bakhtar, J. B. Young, A. White and D. A. Simpson, "Classical nucleation theory and its application to condensing steam flow calculations," J. Mechanical Engineering Science, vol. 219, no. 12, pp. 1315-1333, 2004.

[40] H. M. Hulburt and S. Katz, "Some problems in particle technology: A statistical mechanical formulation," Chemical Engineering Science, vol. 19, no. 8, pp. 555-574, 1964. 